\title{
A Tentative Viewpoint on Presupposition of Invariance of Light Speed
}

\author{
Xinli Zhou \\ Shaoyang University, Shaoyang 422003, China \\ Email: 2745002134@qq.com
}

\begin{abstract}
In order to analyze the presupposition of the invariance of light speed, the actual propagation distance of satellite signals between satellites and ground was measured by GNSS receivers in GNSS measurement experiments. It was found that the actual propagation distance was not the distance between the space position point of the satellite signal at the moment of transmitting, which we usually think of as the satellite ephemeris, and the receiving station, but the distance between the instantaneous satellite position point when the receiver receives the signal and the receiving station. It was concluded that for different reference systems, only by standing in the high-order multi-dimensional space of four or more dimensions, can we correctly understand that the essence of the invariance of light speed, which is actually the same mutual space distance with the same mutual time interval, rather than the different mutual space distance with the mutual different time.
\end{abstract}

Keywords: principle of invariance of light speed, relativistic effect, global positioning system, high-order multidimensional space, invariance of spatial distance.

\section{$1 \quad$ Introduction}

For the speed of light, whether Newton's classical mechanics or Einstein's theory of relativity, both theories presuppose the premise that if a light pulse is emitted from one place in space to another, the space distance traveled by light is inconsistent for different observers in this process (e.g., for two inertial reference objects in relative motion $P$ and $Q$ when a light pulse is emitted everywhere from $P$ at the position $a$ and when the light pulse is received by $Q, P$ already moves at the position $c$, it is generally accepted by physicists either Newton or Einstein that the space distance that light travels in this process is $c b$ for $P$ and $a b$ for $Q$, which is shown in Figure 1).

On this basis, the divergence arises from Newton's theory and relativity theory. Newton's theory believes that different observers have no objection to the time spent on light in this process, so the speed of light propagation will be different for different observers; while relativity theory, based on Michelson's interference experiment, believes that different observers will spend different time for the light propagation on the premise of affirming the constant speed of light ${ }^{[1][2][3][4]}$. However, the author finds that for the problems of Michelson's interference experiment and the time paradox after the establishment of relativity, as well as the disputes and incompatibilities between quantum theory based on relativity and string theory after that, all of them are rooted in the subjective illusion of the presupposed premise in the sense of space distance. The situation is that the space distance and time spent by light in this process should be consistent for different observers or reference frames (that is, the space distance traveled by light in this process should be in fact the same for both observers, either $P$ or $Q$, should be $c b$ )!

The reason is that people overlook the issue that, unlike the speed of motion of particles or other objects, the space where the speed of light travels is not definitive in advance by either observer's frame of reference or any other frame of reference and it is absolutely static three-dimensional space; in other words, any two relative inertial frames of reference or reference, although relative to each other, are moving, both are essentially static for the entire universe or for light. Therefore, when light propagates between the two inertial reference systems or reference objects, the light does not travel through a three-dimensional space distance based on the stationary system as one of the reference systems or reference objects, but travel through a high-order space distance using the whole cosmic space as the background; even if the distance varies with time, it has nothing to do with the selection of the coordinate system ${ }^{[5][6]}$. This also means that 
the starting point of the spatial distance covered by light during this period can not be calculated by the conventional 3-D rectangular coordinates.

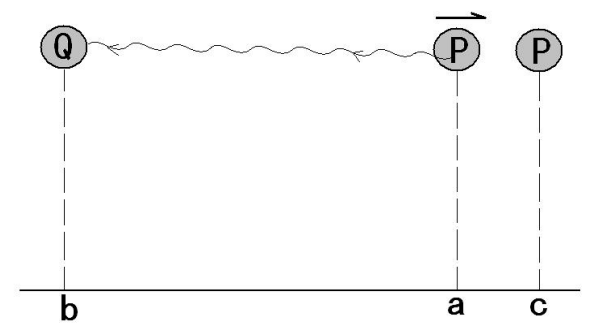

Figure 1. Spatial distance traveled in the process of light pulses being emitted from one part of space to another

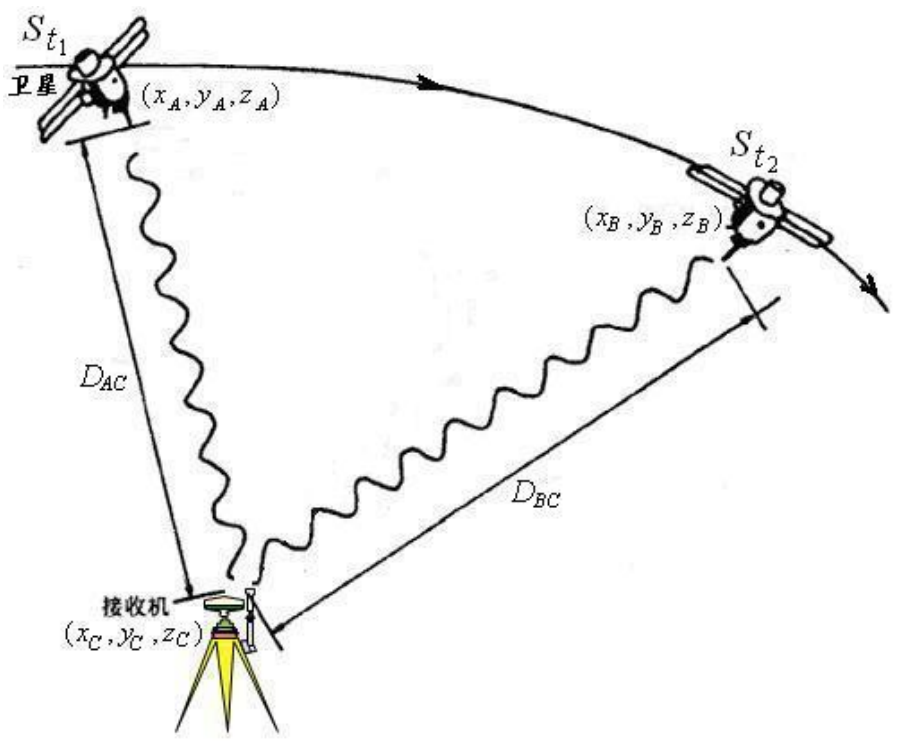

Figure 2. Spatial distance actually propagated by satellite signals

\section{$2 \quad$ Experiments}

In fact, we can judge this by the experiment of GNSS measurement: that is, we assume that a 3-D rectangular coordinate system with the geocentric origin $o$ is established in the geostationary reference system $o-x y z$, and the time of the ground $t$ is used as the time standard. When an earth satellite over the ground $S$ sends out a signal at any time $t_{1}$, the instantaneous position of the satellite is set to $A$; when the GNSS receiver located at the point of ground observation $C$ receives the signal transmitted at $t_{2}$, the satellite has actually moved to the position of $B$ above the ground (as shown in Figure 2). At present, in the process of GNSS precise positioning, the international consensus is to take the 3-D coordinate values of the point $A\left(x_{A}, y_{A}, z_{A}\right)$ as the starting point of the space distance transmitted by the satellite signal during this period to consider the influence of relativistic effect when measuring the point $C\left(x_{C}, y_{C}, z_{C}\right)^{[7][8][9][10][11][12][13]}$. In other words, according to Einstein's special and general relativity theory, when a clock with a frequency of $f_{0}$ on the ground is installed on a satellite running at a certain speed of $v_{S}$, it will generate motion frequency shift and gravitational frequency shift. The total time error resulting from this is assumed to be that the satellite signal is $\Delta t_{S}$ without considering the influence of other factors such as earth rotation, satellite ephemeris error and signal propagation error. The spatial distance propagated during this time " $t_{12}=t_{2}-t_{1}$ " is " $D=c t$ " with the elimination of $\Delta t_{S}$, which is: 


$$
c\left(t_{12}-\Delta t_{S}\right)=\sqrt{\left(x_{C}-x_{A}\right)^{2}+\left(y_{C}-y_{A}\right)^{2}+\left(z_{C}-z_{A}\right)^{2}}
$$

There must be a problem here: if the satellite transmits optical signals from the freely stationary point $A$ over the earth, then there is no objection that the propagation distance of the satellite signal in space for the GNSS receiver located at the observation point $C$ is $D_{A C}$; however, if the satellite transmits optical signals from the point $A$ over the earth where it just arrives instantaneously by free movement, is the propagation distance of the satellite signal in space for the GNSS receiver located at the observation point $C$ still $D_{A C}$ ? This is uncertain! Because this distance is the conclusion that we take the earth as the three-dimensional space of the geostationary system. If we stand in the perspective of satellite, the actual propagation distance of satellite signal measured by GNSS receiver in space is not $D_{A C}$; however, it is the distance between the satellite's position point $B\left(x_{B}, y_{B}, z_{B}\right)$ and the receiver's location point $C$ when the receiver receives the satellite signal! Obviously, if we take the coordinate value of the point as the starting point $B$ and consider the coordinate calculation of the observation point $C$, the results are actually different, which is the following:

$$
\begin{aligned}
c t & =\sqrt{\left(x_{C}-x_{B}\right)^{2}+\left(y_{C}-y_{B}\right)^{2}+\left(z_{C}-z_{B}\right)^{2}} \\
& =\sqrt{\left(x_{C}-\left(x_{A}+x_{A B}\left(t_{A C}\right)\right)\right)^{2}+\left(y_{C}-\left(y_{A}+y_{A B}\left(t_{A C}\right)\right)\right)^{2}+\left(z_{C}-\left(z_{A}+z_{A B}\left(t_{A C}\right)\right)\right)^{2}}
\end{aligned}
$$

In other words, on the premise of considering only the influence of this error factor, assuming the measurement coordinate error of the observation point $C 1$ is $\Delta x_{C 1}, \Delta y_{C 1}, \Delta z_{C 1}$, and the coordinates of the point $C 1\left(x_{C 1}, y_{C 1}, z_{C 1}\right)$ calculated according to the theoretical values are as the following

$$
\left(\begin{array}{l}
x_{C 1} \\
y_{C 1} \\
z_{C 1}
\end{array}\right)=\left(\begin{array}{l}
x_{J 1} \\
y_{J 1} \\
z_{J 1}
\end{array}\right)+\left(\begin{array}{ccc}
\delta_{C 1} & H_{C 1} & -G_{C 1} \\
-H_{C 1} & \delta_{C 1} & L_{C 1} \\
G_{C 1} & -L_{C 1} & \delta_{C 1}
\end{array}\right)\left(\begin{array}{l}
x_{J 2}-x_{J 1} \\
y_{J 2}-y_{J 1} \\
z_{J 2}-z_{J 1}
\end{array}\right)
$$

then, the obtained $\Delta x_{C 1}, \Delta y_{C 1}, \Delta z_{C 1}$ based on the principle of formula (1) are independent variables unrelated to each other and should oscillate up and down on the three coordinate axes of the point $C 1$, because

$$
\left(\begin{array}{l}
\Delta x_{C 1} \\
\Delta y_{C 1} \\
\Delta z_{C 1}
\end{array}\right)=\left(\begin{array}{l}
x_{C 1}^{\prime}-x_{C 1} \\
y_{C 1}^{\prime}-y_{C 1} \\
z_{C 1}^{\prime}-z_{C 1}
\end{array}\right)=\left(\begin{array}{ccc}
\delta_{C 1}^{\prime}-\delta_{C 1} & H_{C 1}^{\prime}-H_{C 1} & -G_{C 1}^{\prime}+G_{C 1} \\
-H_{C 1}^{\prime}+H_{C 1} & \delta_{C 1}^{\prime}-\delta_{C 1} & L_{C 1}^{\prime}-L_{C 1} \\
G_{C 1}^{\prime}-G_{C 1} & -L_{C 1}^{\prime}+L_{C 1} & \delta_{C 1}^{\prime}-\delta_{C 1}
\end{array}\right)\left(\begin{array}{l}
x_{J 2}-x_{J 1} \\
y_{J 2}-y_{J 1} \\
z_{J 2}-z_{J 1}
\end{array}\right)
$$

While, the obtained $\Delta x_{C 1}, \Delta y_{C 1}, \Delta z_{C 1}$ based on the principle of formula (2) are non-independent variables related to each other, and there are obvious systematic errors related to the coordinates on the three axes because the error results are as the following:

$$
\left(\begin{array}{l}
\Delta x_{C 1} \\
\Delta y_{C 1} \\
\Delta z_{C 1}
\end{array}\right)=\left(\begin{array}{l}
x_{J 1}^{t 2}-x_{J 1}^{t 1} \\
y_{J 1}^{t 2}-y_{J 1}^{t 1} \\
z_{J 1}^{t 2}-z_{J 1}^{t 1}
\end{array}\right)+\left(\begin{array}{ccc}
\delta_{C 1}^{t 23} & H_{C 1}^{t 23} & -G_{C 1}^{t 23} \\
-H_{C 1}^{t 2} & \delta_{C 1}^{t 2} & L_{C 1}^{t 2} \\
G_{C 1}^{t 2} & -L_{C 1}^{t 2} & \delta_{C 1}^{t 2}
\end{array}\right)\left(\begin{array}{l}
x_{J 2}^{t 3}-x_{J 1}^{t 2} \\
y_{J 2}^{t 3}-y_{J 1}^{t 2} \\
z_{J 2}^{t 3}-z_{J 1}^{t 2}
\end{array}\right)-\left(\begin{array}{ccc}
\delta_{C 1}^{t 1} & H_{C 1}^{t 1} & -G_{C 1}^{t 1} \\
-H_{C 1}^{t 1} & \delta_{C 1}^{t 1} & L_{C 1}^{t 1} \\
G_{C 1}^{t 1} & -L_{C 1}^{t 1} & \delta_{C 1}^{t 1}
\end{array}\right)\left(\begin{array}{l}
x_{J 2}^{t 1}-x_{J 1}^{t 1} \\
y_{J 2}^{t 1}-y_{J 1}^{t 1} \\
z_{J 2}^{11}-z_{J 1}^{t 1}
\end{array}\right)
$$

However, the actual situation is not as simple as imagined, because the actual measurement results often contain comprehensive errors of many factors. For example, the measurement errors of the above point $C 1$ may include not only the satellite clock error oscillating up and down on the three coordinate axes of the point $C 1$, but also the ephemeris errors related to the three coordinate axes. Namely

$$
\left(\begin{array}{l}
\Delta x_{C 1} \\
\Delta y_{C 1} \\
\Delta z_{C 1}
\end{array}\right)=\left(\begin{array}{l}
x_{J 1}^{\prime}-x_{J 1} \\
y_{J 1}^{\prime}-y_{J 1} \\
z_{J 1}^{\prime}-z_{J 1}
\end{array}\right)+\left(\begin{array}{ccc}
\delta_{C 1}^{\prime \prime} & H_{C 1}^{\prime \prime} & -G_{C 1}^{\prime \prime} \\
-H_{C 1}^{\prime \prime} & \delta_{C 1}^{\prime \prime} & L_{C 1}^{\prime \prime} \\
G_{C 1}^{\prime \prime} & -L_{C 1}^{\prime \prime} & \delta_{C 1}^{\prime \prime}
\end{array}\right)\left(\begin{array}{l}
x_{J 2}^{\prime}-x_{J 1}^{\prime} \\
y_{J 2}^{\prime}-y_{J 1}^{\prime} \\
z_{J 2}^{\prime}-z_{J 1}^{\prime}
\end{array}\right)-\left(\begin{array}{ccc}
\delta_{C 1}^{\prime} & H_{C 1}^{\prime} & -G_{C 1}^{\prime} \\
-H_{C 1}^{\prime} & \delta_{C 1}^{\prime} & L_{C 1}^{\prime} \\
G_{C 1}^{\prime} & -L_{C 1}^{\prime} & \delta_{C 1}^{\prime}
\end{array}\right)\left(\begin{array}{l}
x_{J 2}-x_{J 1} \\
y_{J 2}-y_{J 1} \\
z_{J 2}-z_{J 1}
\end{array}\right)
$$

In this way, how can we effectively distinguish this difference? Here, a relatively simple solution is that if the side length of the two observation points from which two GNSS receivers synchronously measure the satellite signal are compared with the known side length, the difference between the difference mentioned above can be clearly distinguished. Specifically, if assuming that the coordinates of another observation 
point $C 2\left(x_{C 2}, y_{C 2}, z_{C 2}\right)$, which synchronously measures the satellite signal, are calculated according to its theoretical values as the following:

$$
\left(\begin{array}{l}
x_{C 2} \\
y_{C 2} \\
z_{C 2}
\end{array}\right)=\left(\begin{array}{l}
x_{J 1} \\
y_{J 1} \\
z_{J 1}
\end{array}\right)+\left(\begin{array}{ccc}
\delta_{C 2} & H_{C 2} & -G_{C 2} \\
-H_{C 2} & \delta_{C 2} & L_{C 2} \\
G_{C 2} & -L_{C 2} & \delta_{C 2}
\end{array}\right)\left(\begin{array}{l}
x_{J 2}-x_{J 1} \\
y_{J 2}-y_{J 1} \\
z_{J 2}-z_{J 1}
\end{array}\right)
$$

then, the theoretical coordinate difference between the point $C 1$ and the point $C 2$ should be:

$$
\left(\begin{array}{c}
x_{C 2}-x_{C 1} \\
y_{C 2}-y_{C 1} \\
z_{C 2}-z_{C 1}
\end{array}\right)=\left(\begin{array}{ccc}
\delta_{C 2}-\delta_{C 1} & H_{C 2}-H_{C 1} & -G_{C 2}+G_{C 1} \\
-H_{C 2}+H_{C 1} & \delta_{C 2}-\delta_{C 1} & L_{C 2}-L_{C 1} \\
G_{C 2}-G_{C 1} & -L_{C 2}+L_{C 1} & \delta_{C 2}-\delta_{C 1}
\end{array}\right)\left(\begin{array}{l}
x_{J 2}-x_{J 1} \\
y_{J 2}-y_{J 1} \\
z_{J 2}-z_{J 1}
\end{array}\right)
$$

Now, if the principle of formula (1) is followed, the difference of coordinates between these two points is:

$$
\left(\begin{array}{c}
x_{C 2}^{\prime}-x_{C 1}^{\prime} \\
y_{C 2}^{\prime}-y_{C 1}^{\prime} \\
z_{C 2}^{\prime}-z_{C 1}^{\prime}
\end{array}\right)=\left(\begin{array}{ccc}
\delta_{C 2}^{\prime \prime}-\delta_{C 1}^{\prime \prime} & H_{C 2}^{\prime \prime}-H_{C 1}^{\prime \prime} & -G_{C 2}^{\prime \prime}+G_{C 1}^{\prime \prime} \\
-H_{C 2}^{\prime \prime}+H_{C 1}^{\prime \prime} & \delta_{C 2}^{\prime \prime}-\delta_{C 1}^{\prime \prime} & L_{C 2}^{\prime \prime}-L_{C 1}^{\prime \prime} \\
G_{C 2}^{\prime \prime}-G_{C 1}^{\prime \prime} & -L_{C 2}^{\prime \prime}+L_{C 1}^{\prime \prime} & \delta_{C 2}^{\prime \prime}-\delta_{C 1}^{\prime \prime}
\end{array}\right)\left(\begin{array}{l}
x_{J 2}^{\prime}-x_{J 1}^{\prime} \\
y_{J 2}^{\prime}-y_{J 1}^{\prime} \\
z_{J 2}^{\prime}-z_{J 1}^{\prime}
\end{array}\right)
$$

However, if the principle of formula (2) is followed, the difference of coordinates between these two points is:

$$
\left(\begin{array}{l}
x_{C 2}^{\prime}-x_{C 1}^{\prime} \\
y_{C 2}^{\prime}-y_{C 1}^{\prime} \\
z_{C 2}^{\prime}-z_{C 1}^{\prime}
\end{array}\right)=\left(\begin{array}{l}
x_{J 1}^{t 4}-x_{J 1}^{t 2} \\
y_{J 1}^{t 4}-y_{J 1}^{t 2} \\
z_{J 1}^{t 4}-z_{J 1}^{t 2}
\end{array}\right)+\left(\begin{array}{ccc}
\delta_{C 1}^{t 45} & H_{C 1}^{t 45} & -G_{C 1}^{t 45} \\
-H_{C 1}^{t 45} & \delta_{C 1}^{t 45} & L_{C 1}^{t 45} \\
G_{C 1}^{t 45} & -L_{C 1}^{t 45} & \delta_{C 1}^{t 45}
\end{array}\right)\left(\begin{array}{l}
x_{J 2}^{t 5}-x_{J 4}^{t 4} \\
y_{J 2}^{t 5}-y_{J 1}^{t 4} \\
z_{J 2}^{t 5}-z_{J 1}^{t 4}
\end{array}\right)-\left(\begin{array}{ccc}
\delta_{C 1}^{t 23} & H_{C 1}^{t 23} & -G_{C 1}^{t 23} \\
-H_{C 1}^{t 23} & \delta_{C 1}^{t 23} & L_{C 1}^{t 23} \\
G_{C 1}^{t 23} & -L_{C 1}^{t 23} & \delta_{C 1}^{t 23}
\end{array}\right)\left(\begin{array}{l}
x_{J 2}^{t 3}-x_{J 1}^{t 2} \\
y_{J 2}^{t 3}-y_{J 1}^{t 2} \\
z_{J 2}^{t 3}-z_{J 1}^{t 2}
\end{array}\right)
$$

Obviously, when comparing the measured side lengths $s_{i}^{\prime}(i=1,2,3, \cdots)$ with their theoretical values $s_{i}$, we safely draw the conclusion that based on the principle of formula (1), the results have a very small variance, while based on the principle of formula (2), the results have a very big variance!

Next, in order to verify this situation, 49 national first-class traverse points with known Beijing 54 plane coordinates and elevations are selected as the observation points in this experiment in an area near 111 degrees of the central meridian in China.

During the measurement, 49 test machines are used to measure the phase of the same carrier of four or more satellites above the ground at each observation point in the surveying area and at the same observation epoch, respectively, using a dual-frequency geodesic GNSS receiver of Zhonghaida v90 (plane accuracy of static differential positioning is $2.5 \mathrm{~mm}+1 \mathrm{ppm} \times \mathrm{D}$, elevation accuracy is $5 \mathrm{~mm}+1 \mathrm{ppm} \times \mathrm{D}$ and single positioning accuracy is $1.5 \mathrm{~m})$. After observing 16 epochs synchronously and statically, by calculating the known Beijing 54 plane coordinates and elevations of 49 observation points, they are transformed into 3 -D rectangular coordinates in the same ellipsoid space. Then, the set of undifferentiated WGS-84 coordinates of these points measured by GNSS are compared with the set of its known Beijing 54 coordinates, and the coordinate deviation $d_{2 N}(N=1,2, \cdots, 1176)$ of each point and its average $\Delta \bar{x}, \Delta \bar{y}, \Delta \bar{z}$, variance $\sigma_{x}, \sigma_{y}, \sigma_{z}$ and covariance $\sigma_{x y}, \sigma_{y z}, \sigma_{z x}$ are obtained. At the same time, the side lengths and its coordinate-difference of $\frac{1}{2} \times 49 \times(49-1)=1176$ baseline vectors between observation points calculated by GNSS coordinates is compared with the length and the coordinate-difference of baseline vectors calculated by known coordinates, and the deviations of the lengths $d_{N}(N=1,2, \ldots, 1176)$ of each baseline vectors and its average value $\bar{d}$, variance $\sigma_{d}$ are calculated; moreover, the deviations of the coordinate-difference $d \Delta x_{N}, d \Delta y_{N}, d \Delta z_{N}$ of each baseline vectors and its variance $\sigma_{d x}, \sigma_{d y}, \sigma_{d z}$, covariance $\sigma_{d x y}, \sigma_{d y z}, \sigma_{d z x}$ are obtained.

\section{$3 \quad$ Results and Discussion}

As can be seen from the table 1:

$$
\sigma_{d x}, \sigma_{d y}, \sigma_{d z} \rightarrow 2 \sigma_{x}, 2 \sigma_{y}, 2 \sigma_{z}, \bar{d} \rightarrow 0
$$


It showed that there are obvious systematic errors related to coordinates in the three coordinate axes of GNSS measurement. In order to further understand the details, the GNSS measurement data are transformed through rigorous coordinates transformation, and the rigorous adjustment is carried out in the following two different ways:

Table 1. The comparison of the measured coordinates of GNSS with the known coordinates

\begin{tabular}{|c|c|c|c|c|c|c|c|c|c|c|c|}
\hline \multirow[t]{2}{*}{$\begin{array}{l}\text { Measuring } \\
\text { point }\end{array}$} & \multicolumn{3}{|c|}{$\begin{array}{l}\text { The difference between } \\
\text { the measured } \\
\text { coordinates and the } \\
\text { known coordinates }(\mathrm{m})\end{array}$} & \multirow[t]{2}{*}{$\begin{array}{l}\text { Measuring } \\
\text { point }\end{array}$} & \multicolumn{3}{|c|}{$\begin{array}{c}\text { The difference between } \\
\text { the measured coordinates } \\
\text { and the known } \\
\text { coordinates }(\mathrm{m})\end{array}$} & \multirow[t]{2}{*}{$\begin{array}{l}\text { Measuring } \\
\text { point }\end{array}$} & \multicolumn{3}{|c|}{$\begin{array}{l}\text { The difference between } \\
\text { the measured } \\
\text { coordinates and the } \\
\text { known coordinates }(\mathrm{m})\end{array}$} \\
\hline & $\Delta x$ & $\Delta y$ & $\Delta z$ & & $\Delta x$ & $\Delta y$ & $\Delta z$ & & $\Delta x$ & $\Delta y$ & $\Delta z$ \\
\hline 01 & -4.5 & -133 & -63.25 & 18 & -3.5 & -131.5 & -66.75 & 35 & -1.875 & -130.5 & -67.75 \\
\hline 02 & -2.125 & -136 & -66 & 19 & -3.5 & -132 & -66.75 & 36 & -1.875 & -130.5 & -67.5 \\
\hline 03 & -2.125 & -136 & -66 & 20 & -3.5 & -132 & -67 & 37 & -3.625 & -133.5 & -64.75 \\
\hline 04 & -2.125 & -136 & -66 & 21 & -4.375 & -130 & -69.25 & 38 & -3.625 & -133.5 & -64.75 \\
\hline 05 & -10.25 & -127 & -52.75 & 22 & -4.5 & -130 & -69.25 & 39 & -3.625 & -133.5 & -64.75 \\
\hline 06 & -10.25 & -127.5 & -52.75 & 23 & -3.625 & -132 & -66.75 & 40 & -3.75 & -133.5 & -64.75 \\
\hline 07 & -10.25 & -127.5 & -52.75 & 24 & -3.625 & -132 & -66.75 & 41 & -3.625 & -133 & -64.75 \\
\hline 08 & -10.25 & -127.5 & -52.75 & 25 & -5.625 & -132.5 & -63 & 42 & -3.625 & -133.5 & -64.75 \\
\hline 09 & -10.25 & -127.5 & -52.75 & 26 & -5.625 & -133 & -63.25 & 43 & -3.625 & -133.5 & -64.75 \\
\hline 10 & -4.5 & -130 & -69.5 & 27 & -2.75 & -132.5 & -67.5 & 44 & -3.625 & -133 & -65 \\
\hline 11 & -4.375 & -130 & -69.25 & 28 & -5.625 & -132.5 & -63.25 & 45 & -3.625 & -133 & -65 \\
\hline 12 & -2.5 & -140.5 & -59.75 & 29 & -5.625 & -133 & -63.25 & 46 & -4.5 & -132.5 & -63 \\
\hline 13 & -2.5 & -140 & -59.5 & 30 & -5.75 & -133 & -63.25 & 47 & -3.625 & -140 & -59.5 \\
\hline 14 & -2.375 & -140.5 & -59.75 & 31 & -5.75 & -132.5 & -63.25 & 48 & -3.5 & -140 & -59.75 \\
\hline 15 & -2.5 & -140.5 & -59.5 & 32 & -4.375 & -131 & -58.25 & 49 & -3.625 & -140 & -59.5 \\
\hline 16 & -10.25 & -127.5 & -53 & 33 & -4.375 & -131 & -58.25 & & & & \\
\hline 17 & -10.25 & -127.5 & -53 & 34 & -4.5 & -131 & -58.25 & & & & \\
\hline \multicolumn{2}{|c|}{$\begin{array}{c}\text { The precision } \\
\text { statistics }\end{array}$} & & & $\begin{array}{r}\Delta \bar{x}= \\
\sigma_{x}= \\
\sigma_{x y} \\
\sigma_{d}=23.561 \mathrm{~m} \\
\bar{d}=-1.233 \mathrm{~m}\end{array}$ & $\begin{aligned} &-4.689 n \\
&= 6.291 \mathrm{~m} \\
&=-6.27 \mathrm{n} \\
&, \sigma_{d x}= \\
& \sigma_{d x y}=\end{aligned}$ & $\begin{array}{c}\quad \Delta \bar{y}= \\
\sigma_{y}=1 \\
, \quad \sigma_{y z}= \\
2.581 m, \\
12.8 m,\end{array}$ & $\begin{array}{l}-132.8 m, \\
.117 m, \\
3.28 m, \\
\sigma_{d y}=28 \\
\sigma_{d y z}=6.6\end{array}$ & $\begin{array}{c}\Delta \bar{z}=-62.5 r \\
\sigma_{z}=25.271 m \\
\sigma_{z x}=-9.24 m \\
.239 m, \quad \sigma_{d z}= \\
88 m, \quad \sigma_{d z x}=\end{array}$ & $\begin{array}{l}=50.542 \mathrm{~m} \\
-18.866 \mathrm{~m}\end{array}$ & & \\
\hline
\end{tabular}

The first method is based on the difference technology commonly used in GNSS data, that is, the one-time difference between stations and epochs is mainly between two stations and two epochs, which means that 47 synchronous observations in this experiment are redundant observations, while 14 of 16 observations in each station are processed according to redundant observations. In this way, the difference equation between the two stations should be as follows, by considering only the elimination of relativistic effect:

$$
\left\{\begin{array}{l}
c\left(t_{1}-\left(t_{0}+\Delta t_{S}\right)\right)=\sqrt{\left(x_{1}-x_{t_{0}}\right)^{2}+\left(y_{1}-y_{t_{0}}\right)^{2}+\left(z_{1}-z_{t_{0}}\right)^{2}} \\
c\left(t_{2}-\left(t_{0}+\Delta t_{S}\right)\right)=\sqrt{\left(x_{2}-x_{t_{0}}\right)^{2}+\left(y_{2}-y_{t_{0}}\right)^{2}+\left(z_{2}-z_{t_{0}}\right)^{2}}
\end{array}\right.
$$

In the formula:

$x_{t_{0}}, y_{t_{0}}, z_{t_{0}}-$-The position coordinates of satellites in space obtained from the ephemeris of satellites;

$x_{i}, y_{i}, z_{i}(i=1,2, \cdots, 227)$--GNSS measurements at observation point $i$;

$\Delta t_{S}$--Satellite clock error caused by relativistic effect.

While, the difference equation between the two epochs should be: 


$$
\left\{\begin{array}{l}
c\left(t_{1}-\left(t_{0}+\Delta t_{S}\right)\right)=\sqrt{\left(x_{i}-x_{t_{0}}\right)^{2}+\left(y_{i}-y_{t_{0}}\right)^{2}+\left(z_{i}-z_{t_{0}}\right)^{2}} \\
c\left(t_{2}-\left(t_{1}+\Delta t_{S}\right)\right)=\sqrt{\left(x_{i}-x_{t_{1}}\right)^{2}+\left(y_{i}-y_{t_{1}}\right)^{2}+\left(z_{i}-z_{t_{1}}\right)^{2}}
\end{array}\right.
$$

The second method is to take into account that the $3-\mathrm{D}$ coordinate value of the satellite ephemeris at the point of "A" as the starting point of GNSS receiver ranging, and the measurement result is likely to have gross error related to the propagation time $t$ of satellite signals in the three coordinate axes of the satellite $((\Delta x(t), \Delta y(t), \Delta z(t)))$. If assuming that this gross error is a second-order polynomial with six unknowns, that is:

$$
\left(\begin{array}{l}
\Delta x(t) \\
\Delta y(t) \\
\Delta z(t)
\end{array}\right)=t\left(\begin{array}{l}
x^{(1)} \\
y^{(1)} \\
z^{(1)}
\end{array}\right)+\frac{1}{2} t^{2}\left(\begin{array}{l}
x^{(2)} \\
y^{(2)} \\
z^{(2)}
\end{array}\right)
$$

Then, the difference technology used in this method needs to make a difference between at least seven stations and seven epochs in order to eliminate these six gross error unknowns $\left(x^{(1)}, y^{(1)}, z^{(1)}, x^{(2)}, y^{(2)}, z^{(2)}\right)$. In other words, only 42 synchronous observations in this experiment are redundant observations, and only 9 of 16 observations in each station are processed according to redundant observations. Therefore, in this way, the difference equation between the seven stations should be as the followings, only considering the effect of eliminating the gross errors of satellite coordinates:

$$
\left\{\begin{array}{l}
c t_{1}=\sqrt{\left(x_{1}-\left(x_{t_{0}}+\Delta x\left(t_{1}\right)\right)\right)^{2}+\left(y_{1}-\left(y_{t_{0}}+\Delta y\left(t_{1}\right)\right)\right)^{2}+\left(z_{1}-\left(z_{t_{0}}+\Delta z\left(t_{1}\right)\right)\right)^{2}} \\
c t_{2}=\sqrt{\left(x_{2}-\left(x_{t_{0}}+\Delta x\left(t_{2}\right)\right)\right)^{2}+\left(y_{2}-\left(y_{t_{0}}+\Delta y\left(t_{2}\right)\right)\right)^{2}+\left(z_{2}-\left(z_{t_{0}}+\Delta z\left(t_{2}\right)\right)\right)^{2}} \\
\cdots \cdots \cdots \cdots \cdots \cdots \cdots \cdots \cdots \cdots \cdots \cdots \cdots \cdots \cdots \cdots \cdots \\
c t_{7}=\sqrt{\left.\left(x_{7}-\left(x_{t_{0}}+\Delta x\left(t_{7}\right)\right)\right)^{2}+\left(y_{7}-\left(y_{t_{0}}+\Delta y t_{7}\right)\right)\right)^{2}+\left(z_{7}-\left(z_{t_{0}}+\Delta z\left(t_{7}\right)\right)\right)^{2}}
\end{array}\right.
$$

The difference equation between the seven epochs should be:

$$
\left\{\begin{array}{l}
c \Delta t_{1}=\sqrt{\left(x_{i}-\left(x_{t_{0}}+\Delta x\left(t_{1}\right)\right)\right)^{2}+\left(y_{i}-\left(y_{t_{0}}+\Delta y\left(t_{1}\right)\right)\right)^{2}+\left(z_{i}-\left(z_{t_{0}}+\Delta z\left(t_{1}\right)\right)\right)^{2}} \\
c \Delta t_{2}=\sqrt{\left(x_{i}-\left(x_{t_{0}}+\Delta x\left(t_{2}\right)\right)\right)^{2}+\left(y_{i}-\left(y_{t_{0}}+\Delta y\left(t_{2}\right)\right)\right)^{2}+\left(z_{i}-\left(z_{t_{0}}+\Delta z\left(t_{2}\right)\right)\right)^{2}} \\
\cdots \cdots \cdots \cdots \cdots \cdots \cdots \cdots \cdots \cdots \cdots \cdots \cdots \cdots \cdots \cdots \cdots \\
c \Delta t_{7}=\sqrt{\left(x_{i}-\left(x_{t_{0}}+\Delta x\left(t_{7}\right)\right)\right)^{2}+\left(y_{i}-\left(y_{t_{0}}+\Delta y\left(t_{7}\right)\right)\right)^{2}+\left(z_{i}-\left(z_{t_{0}}+\Delta z\left(t_{7}\right)\right)\right)^{2}}
\end{array}\right.
$$

In the formula:

$$
\Delta t_{i}=t_{i}-t_{i-1} \quad(i=1,2, \cdots, 16)
$$

The results were combined with the first method and the second method showing that the second method is obviously better in the reconstruction accuracy than the first method, neither on the precision $\left(m_{x}, m_{y}, m_{z}\right)$ in the coordinates of the observation points nor the precision $\left(m_{d}\right)$ in the coordinate difference of baseline vectors, namely ${ }^{[19][20]}$ :

$$
\begin{gathered}
m_{x 2}, m_{y 2}, m_{z 2}<m_{x 1}, m_{y 1}, m_{z 1}, \text { and } m_{d 2}<m_{d 1} \\
m_{d x 1}, m_{d y 1}, m_{d z 1} \rightarrow 3 m_{x 1}, 3 m_{y 1}, 3 m_{z 1} \text {, and } m_{d x 2}, m_{d y 2}, m_{d z 2} \rightarrow m_{x 2}, m_{y 2}, m_{z 2}
\end{gathered}
$$

This demonstrates that for GNSS ranging, there must be the coordinate deviation on the directions of three coordinate axes, which can not be completely solved with the current differential technology and are relevant and independent to each other! The fundamental reason for this result is that the propagation distance of satellite signal measured by GNSS receiver is not actually the distance between the point of 
space where the satellite signal is transmitted at the moment and the point of position where the receiver is located, but the distance between the point of space where the satellite moves at the moment when the receiver receives the signal and the point of position where the receiver is located. This distance is not actually a 3-D space distance, but a real-time high-order multi-dimensional space distance. It mainly consists of two parts: one is the 3-D space distance $D_{A C}$, which is between the point in the stationary space " $A$ " that the satellite is passing when it transmits the signal and the observation point " $C$ "; the other is the hyperspace distance $\Delta D_{A}=A^{\prime}-A$, which is the distance difference between the 3-D space distance of the stationary system on the ground between the point " $A$ " and the point " $C$ " when the satellite transmits the signal and the 3-D space of the motion system of the satellite " $S$ ". This distance varies with time, i.e.

$$
\Delta D_{A}=D_{A B}(t)
$$

Assuming that the three-dimensional space of the stationary system where the point " $A$ " and the point " $C$ " are located and the motion system where the satellite " $S$ " is located are two parallel two-dimensional planes, as shown in Fig. 3, it is not difficult to understand that when the satellite transmits signals from the point " $A$ ", the satellite is actually not at the position of the point " $A$ " in the three-dimensional space which is the stationary system as the earth at this moment, but at the orbital position which is located in one of the another higher-order multi-dimensional spaces parallel to the three-dimensional space of the stationary system in the whole universe and appearing to be moving for the stationary system. The orbital coordinates of this point have definitive values $\left(\Delta x_{A}(t), \Delta y_{A}(t), \Delta z_{A}(t)\right)$ in the three coordinate axes corresponding to the position of the point " $A$ ". The orbital coordinates depend on the space order " $n$ " of the orbital point where the satellite is located, and are independent of the quality, size and shape of the satellite. For example, assuming that the space order of the orbital point of the satellite is $50(n=50)$, the 50 -order partial derivation of the satellite for the time " $t$ " in three coordinate axes will be essentially a constant, that is:

$$
\frac{\partial^{50} \Delta x_{A}}{\partial t^{50}}=C_{x}, \frac{\partial^{50} \Delta y_{A}}{\partial t^{50}}=C_{y}, \frac{\partial^{50} \Delta z_{A}}{\partial t^{50}}=C_{z}
$$

Therefore, to be exact, if the space order of the satellite orbital points is 50 , the difference between stations or epochs should be calculated at least once between 151 stations or 151 epochs. Thus, only 151 stations or 151 epochs in the synchronous loop are redundant observations in GNSS data processing. This also means that when we use light to measure the distance between any two reference objects or motion systems in space, the coordinate transformation relationship established between the two reference objects or motion systems can neither be Galileo or Newton transformation based on "velocity relative" nor Lorentz transformation or Einstein transformation based on "time relative", but should be a more rigorous coordinate transformation relationship based on the absolute space distance. Here, the so-called concept of "absolute spatial distance" includes, on one hand, that the spatial distance between any two relatively static points in space remains an absolutely constant value, that is, it is the same fixed value for any reference system and does not change with time; on the other hand, that the spatial distance change between any two relatively uniform or uniformly accelerated motion and $n$ order uniformly accelerated motion keeps an absolute constant ratio, that is, the ratio is the same for any reference frame at any time, which is:

$$
\left\{\begin{array}{l}
x^{\prime} \\
y^{\prime} \\
z^{\prime} \\
t^{\prime}
\end{array}\right\} \neq\left\{\begin{array}{l}
x-v t \\
y \\
z \\
t
\end{array}\right\} \neq\left\{\begin{array}{l}
\frac{x-v t}{1-\frac{v^{2}}{c^{2}}} \\
y \\
z \\
t-\frac{v}{c^{2}} x \\
\sqrt{1-\frac{v^{2}}{c^{2}}}
\end{array}\right\}=\left\{\begin{array}{l}
x-\Delta x(t) \\
y-\Delta y(t) \\
z-\Delta z(t) \\
t
\end{array}\right\}
$$

Inside, 


$$
\left\{\begin{array}{l}
\Delta x(t)=\left(x_{1}^{(1)} t\right)+\left(x_{2}^{(1)} t+\frac{1}{2} x_{2}^{(1)} t^{2}\right)+\cdots+\left(\sum_{i=1}^{n} \frac{1}{i !} x_{n}^{(i)} t^{i}\right) \\
\Delta y(t)=\left(y_{1}^{(1)} t\right)+\left(y_{2}^{(1)} t+\frac{1}{2} y_{2}^{(1)} t^{2}\right)+\cdots+\left(\sum_{i=1}^{n} \frac{1}{i !} y_{n}^{(i)} t^{i}\right) \\
\Delta z(t)=\left(z_{1}^{(1)} t\right)+\left(z_{2}^{(1)} t+\frac{1}{2} z_{2}^{(1)} t^{2}\right)+\cdots+\left(\sum_{i=1}^{n} \frac{1}{i !} z_{n}^{(i)} t^{i}\right)
\end{array}\right.
$$

It is worth mentioning that, according to this principle, if more than $N=3 \times \frac{1}{2} \times 51 \times(51+1)=3978$

fixed stations with known coordinates are selected on the ground and one GNSS receiver is installed in each station to observe the satellite synchronously and statically in the experiment, then we can use the space distance rendezvous method (except that the space distance rendezvous is obviously not the distance rendezvous in the three-dimensional space we usually understand, but is higher than 3978-dimensional space rendezvous) to accurately calculate the actual orbit of the satellite $S$ in space, and this measurement result has naturally excluded the so-called "multi-body problem", "earth rotation" and "relativistic effect" and other factors ${ }^{[14][15][16][17][18]}$, its accuracy does not drift with time!

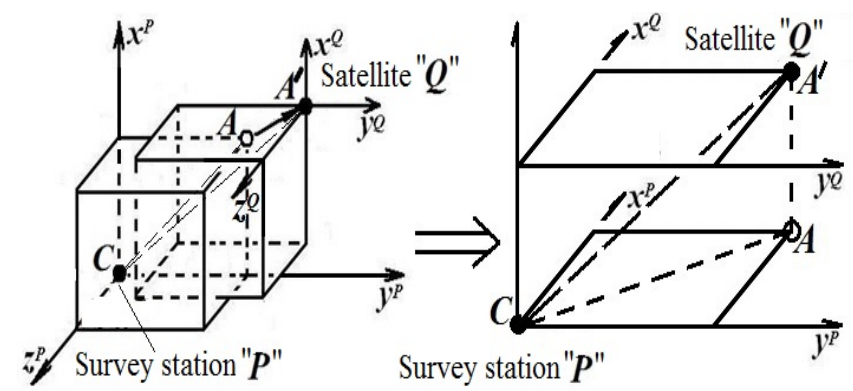

Figure 3. The actual location of the satellite when transmitting signals

\section{Summary}

It can be concluded that it is a misunderstanding to think that the Michelson interference experiment is in conflict with the aberration phenomenon discovered by Bradley in the past. Because the aberration of light is a phenomenon observed when light travels between the two motion systems of the luminous star and the earth. The light source and the receiving point are located in the three-dimensional space of the two different reference systems of the luminous star and the earth respectively. The space distance between the light source and the receiving point essentially represents two high-order multi-dimensional spaces of four or more dimensions and the distance between points varies with time due to the relative motion between the light source and the receiving point. On the contrary, interference experiments are carried out between several relatively stationary points on the interferometer with the earth's motion, all of which are in the same three-dimensional space with the earth as the reference frame. No matter how the earth moves in the universe, the distance between the light source point and each receiving point always represents one of the two points in a three-dimensional space. On this premise, even if the earth itself rotates and revolves around the sun, any distance experienced by light on the interferometer (whether parallel to the direction of the earth's motion or perpendicular to the direction of the earth's motion) will undergo the same time variation.

Therefore, the essence of the so-called "speed invariance of light" is not the result of "different distances for different times", but the result of "the same distance for the same time". In other words, the relativistic effect is essentially not "the relative inconsistency of time", but "the unity of opposites of the absolute consistency of space distance and the relative inconsistency with time change". 


\section{References}

1. Einstein Albert. Relativity [M]. New York: Crown, 1961.

2. Einstein Albert. The Meaning of Relativity [M]. Princeton: Princeton University Press, 1988.

3. Hawking Stephen. A Brief History of Time [M]. New York: Bantam Books, 1988.

4. Thorne Kip. Black Holes and Time Warps [M]. New York: Norton, 1994.

5. Xinli Zhou. Study on the Relativity Effect to GPS Measure Result[J]. Wuhan: Journal of Geomatics, 2013, (1):27-29.

6. Xinli Zhou. Exploring Many-body Problem of the Satellite's Perturbation Equation through Space Dimensionality[J]. Wuhan: Journal of Navigation and Positioning, 2014, 2(1): 1-5.

7. Shaoquan Xu. Principle and Application of GNSS Measurement [M]. Hubei: Wuhan University Press, 2001.

8. Xuesen Li, Lantao Wang, Shaoyuan Li. Relativistic effects and their effects on GNSS technology [J]. Surveying and Mapping Science, 1998, (1): 32-38.

9. Mengyang Zhang, Baowei Lv, Song Wenmiao Song. The effect of relativistic effect on the accuracy of GNSS single point positioning [J]. Journal of Electronics and Information, 1998, 20(5): 663-668.

10.Min Yao, Shengyuan Zhu, Rongshi Pan et al. Relativistic effects of artificial earth satellites in geocentric system [J]. Journal of Astronomy, 1988, 29 (2): 181-189.

11. Neil Ashby. Relativity and the global positioning system [J]. Physics Today, 2002 (5): 41.

12. Hanwei Zhang, Yong Zheng, Lan Du. Relativistic Delay Model for VLBI Observation of Artificial Earth Satellite [J]. Journal of Surveying and Mapping, 2003, (02).

13. Baojun Fei, Zhenghong Chen. More study on the relativistic effect of GNSS [J]. Journal of Armored Forces Engineering College, 2003, 17(2): 1-3.

14. Qien Li. The study on the incompatibility between superluminal speed and relativity [J]. Guangzhou: Journal of Jinan University, 1980, (02).

15. Di Hua. A fundamental revision of Einstein's theory of relativity: relativistic mechanics with variable speed of light (Part I) [J]. Frontier Science, 2009, (04).

16. Di Hua. A fundamental revision of Einstein's theory of relativity: relativistic mechanics with variable speed of light (Part II) [J]. Frontier Science, 2010, (01).

17. Allen D W, et al. Around-the-world Relativistic Sagnac experiment[J]. Science, 1985, 228(4695): 69-70.

18. Ruyong Wang. Extended Sagnac effect, GNSS and the experimental test of two principles of special relativity [J]. Journal of Beijing Institute of Petroleum and Chemical Technology, 2009, 17(1): 53-57.

19. Xinli Zhou. Method for calculating coordinate transformation parameters of navigation GPS[J]. Wuhan: Journal of Geodesy and Geodynamics, 2007, 27(6):68-71.

20. Xinli Zhou. Approach to improve navigation GPS position accuracy[J]. Beijing: Science of Surveying and Mapping, 2008, 33(3): 87-89. 